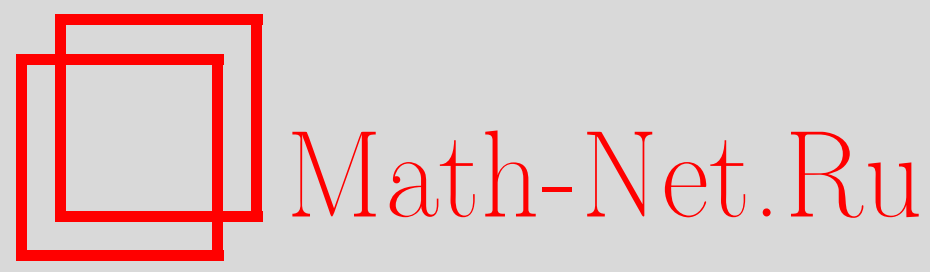

Л. С. Пулькина, Нелокальная задача с интегральными условиями для квазилинейного гиперболического уравнения, Матем. заметки, 2001, том 70, выпуск 1, 88-95

DOI: https://doi.org/10.4213/mzm722

Использование Общероссийского математического портала Math-Net.Ru подразумевает, что вы прочитали и согласны с пользовательским соглашением http://www.mathnet.ru/rus/agreement

Параметры загрузки:

IP : 54.196 .121 .252

26 апреля 2023 г., 18:13:49

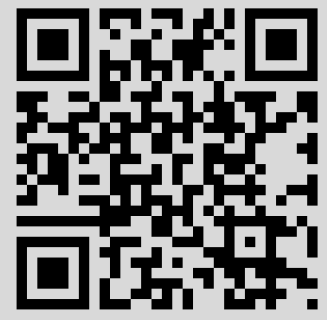




\title{
НЕЛОКАЛЬНАЯ ЗАДАЧА С ИНТЕГРАЛЬНЫМИ УСЛОВИЯМИ ДЛЯ КВАЗИЛИНЕЙНОГО ГИПЕРБОЛИЧЕСКОГО УРАВНЕНИЯ
}

\author{
Л. С. Пулькина
}

\begin{abstract}
В статье рассматривается нелокальная задача с интегральньми условиями для квазилинейного гиперболического уравнения в прямоугольной области. Доказано существование и единственность обобщенного решения.

Библиографой: 20 названий.
\end{abstract}

1. Введение. Математическое моделирование некоторых физических явлений и биологических процессов часто приводит к нелокальным задачам для уравнений в частных производных второго порядка. Весьма удобньм способом описания налагаемых на искомое решение условий является задание их в интегральной форме как среднее значение решения на принадлежаших области, в которой ищется решение, многообразиях. Интегральные условия возникают в тех случаях, когда, например, невозможно непосредственное измерение каких-либо физических величин, но известно их усредненное значение. Подобные ситуации имеют место при изучении явлений, происходящих в плазме [1], процессов распространения тепла [2]-[6], некоторых технологических процессов [7], процессов влагопереноса в пористых средах [8], [9], обратных задачах [10], а также в задачах математической биологии [11] и демографии [12].

По-видимому, одной из первых работ, относящихся к исследованию задач с интегральными условиями, является работа [4], в которой доказана однозначная разрешимость смешанной задачи с условиями Дирихле на части границы и интегральным условием для одномерного уравнения теплопроводности. В статье [2] этот результат распространен на общее уравнение параболического типа.

В настоящее время теория нелокальных задач для дифференщиальных уравнений интенсивно развивается. В работах А. К. Гущина и В. П. Михайлова (см. [13] и библиография там же) исследована разрешимость нелокальных задач для эллиптических уравнений второго порядка, в которьх значение решения на границе рассматриваемой области связано со значениями во внутренних точках с помощью некоторого оператора, которьй, в частности, может быть интегральным. Б.П. Панеях в работе [14] рассмотрел класс нелокальных условий, с помошью которых значения решения в точке гранищы выражаются через интеграл от решения по соответствующей этой точке мере и найдены ограничения, при которых существует единственное классическое решение поставленной задачи. В ряде работ изучено расположение спектра операторов, возникающих при исследовании нелокальных задач для обькновенных дифференциальных уравнений [15] 
и уравнений в частных производных [16]. В последнее время появились работы, в которых изучаются нелокальные задачи с интегральными условиями для гиперболических уравнений. В работах [17], [18] рассмотрены смешанные задачи, в которых одно или оба граничных условия заменены интегральными. В [19] доказана однозначная разрешимость задачи, имеющей в качестве данных только интегральные условия. Следует отметить, что классическое решение задачи в такой постановке, которая может быть названа интегральньм аналогом задачи Гурса, для простейшего уравнения $u_{x y}=0$ получена в [20].

В предлагаемой заметке рассматривается квазилинейное уравнение гиперболического типа, для которого изучается задача с интегральными условиями.

2. Постановка задачи. Рассмотрим уравнение

$$
L u \equiv u_{x y}+(A u)_{x}+(B u)_{y}+C u=f(x, y, u)
$$

коэффициенты которого $A(x, y), B(x, y), C(x, y)$ ограничены, имеют ограниченные производные первого порядка, а коэффициент $C(x, y)$ имеет также ограниченную смешанную производную в прямоугольнике $D=\{(x, y): 0<x<a, 0<y<b\}$. Нас будет интересовать вопрос о существовании и единственности обобщенного решения уравнения (1), удовлетворяющего условиям

$$
\int_{0}^{a} u(x, y) d x=\psi(y), \quad \int_{0}^{b} u(x, y) d y=\phi(x) .
$$

Без ограничения общности можно положить $\psi(y)=\phi(x)=0$. Действительно, заметив, что данные функции должны удовлетворять условию согласования

$$
\int_{0}^{a} \phi(x) d x=\int_{0}^{b} \psi(y) d y
$$

и построив функцию

$$
K(x, y)=\frac{1}{a} \psi(y)+\frac{1}{b} \phi(x)-\frac{1}{a b} \int_{0}^{a} \phi(x) d x,
$$

можно свести поставленную задачу к задаче для уравнения $L \bar{u}=\bar{f}(x, y, \bar{u})$, где $\bar{u}=$ $u-K(x, y), \bar{f}=f-L K$, с однородными интегральными условиями. В дальнейшем будем считать, что $\phi(x)=\psi(y)=0$ и интегральные условия для уравнения (1) имеют вид

$$
\int_{0}^{a} u(x, y) d x=0, \quad \int_{0}^{b} u(x, y) d y=0
$$

Обозначим

$$
U=\left\{u(x, y) \mid u \in C^{1}(D), u_{x y} \in C(D), \int_{0}^{a} u(x, y) d x=0, \int_{0}^{b} u(x, y) d y=0\right\} .
$$

Замыкание этого пространства по норме $\|u\|_{1}^{2}=\iint_{D}\left(u^{2}+u_{x}^{2}+u_{y}^{2}\right) d x d y$ обозначим $\widetilde{H}^{1}(D)$. Таким образом, $\widetilde{H}^{1}(D)$ - гильбертово пространство со скалярным произведением

$$
(u, v)_{1}=\int_{0}^{b} \int_{0}^{a}\left(u v+u_{x} v_{x}+u_{y} v_{y}\right) d x d y
$$


элементами которого являются функции, принадлежащие $H^{1}(D)$ и удовлетворяющие условиям (2). Рассмотрим оператор $l: \widetilde{H}^{1} \rightarrow L_{2}$,

$$
l v=\int_{0}^{y} \int_{0}^{x} v(t, \tau) d t d \tau-\int_{0}^{y} v_{x}(x, \tau) d \tau-\int_{0}^{x} v_{y}(t, y) d t
$$

Введем понятие обобщенного решения задачи $(1),(2)$, следуя обычной процедуре. Предполагая, что $u(x, y)$ - решение поставленной задачи из $U$, умножим обе части равенства $L u=f$ на $l v$ и проинтегрируем по области $D$. Получим равенство скалярных произведений $(L u, l v)_{0}=(f, l v)_{0}$. Рассмотрим скалярное произведение $(L u, l v)_{0}$. Преобразуем его, интегрируя по частям и учитьвая, что $v \in \widetilde{H}^{1}(D)$, а следовательно,

$$
\int_{0}^{a} v(x, y) d x=\int_{0}^{b} v(x, y) d y=0
$$

Выражение, полученное в результате этих преобразований, обозначим $B(u, v)$. Таким образом, имеем

$$
\begin{aligned}
B(u, v)= & (u, v)_{1}+\int_{0}^{b} \int_{0}^{a}\left(A u\left(v_{y}-\int_{0}^{y} v(x, \tau) d \tau\right)+B u\left(v_{x} \int_{0}^{x} v(t, y) d t\right)\right. \\
& \left.-(A u)_{x} \int_{0}^{y} v_{x}(x, \tau) d \tau-(B u)_{y} \int_{0}^{x} v_{y}(t, y) d t\right) d x d y+(C u, l v)_{0} .
\end{aligned}
$$

ОПРЕДЕЛЕНИЕ. Обобщенным решением задачи $(1),(2)$ будем называть функцию $u(x, y) \in \widetilde{H}^{1}(D)$, удовлетворяющую для любой $v(x, y) \in \widetilde{H}^{1}(D)$ тождеству

$$
B(u, v)=(f, l v)_{L_{2}}
$$

3. Существование и единственность решения линейной задачи. Пусть $f(x, y, u)=F(x, y)$. Рассмотрим задачу с условиями (2) для уравнения

$$
L u=F(x, y)
$$

ЛЕммА 1. Если коэффициенты уравнения (4) ограничены, причем $|A| \leqslant M<1$, $|B| \leqslant M<1$, и удовлетворяют неравенствам

$C_{x y} \geqslant 0, \quad A_{y} B_{x}-C^{2} \geqslant 0, \quad \frac{M}{2} A_{y}-\left(A_{x}+C\right)^{2} \geqslant 0, \quad \frac{M}{2} B_{x}-\left(B_{y}+C\right)^{2} \geqslant 0$,

mo

$$
\|u\|_{1}^{2} \leqslant c_{1} B(u, u)
$$

әде $c_{1}=m^{-1}, m=1-M$. 
ДокаЗАтеЛЬСтво. Рассмотрим разность $B(u, u)-m\|u\|_{1}^{2}$. Имеем

$$
\begin{aligned}
& B(u, u)-\|u\|_{1}^{2}=\int_{0}^{b} \int_{0}^{a}\left((1-m)\left(u^{2}+u_{x}^{2}+u_{y}^{2}\right)+A u u_{y}+B u u_{x}\right. \\
& \quad-A u \int_{0}^{y} u(x, \tau) d \tau-B u \int_{0}^{x} u(t, y) d t-A_{x} \int_{0}^{y} u_{x}(x, \tau) d \tau-A u_{x} \int_{0}^{y} u_{x}(x, \tau) d \tau \\
& \left.\quad-B_{y} u \int_{0}^{x} u_{y}(t, y) d t-B u_{y} \int_{0}^{x} u_{y}(t, y) d t\right) d x d y+\int_{0}^{b} \int_{0}^{a} C u l u d x d y
\end{aligned}
$$

Некоторые слагаемыев правой части преобразуем интегрированием по частям, после чего получим

$$
\begin{gathered}
-\int_{0}^{b} \int_{0}^{a} A(x, y) u(x, y) \int_{0}^{y} u(x . \tau) d \tau d x d y=\frac{1}{2} \int_{0}^{b} \int_{0}^{a} A_{y}(x, y)\left(\int_{0}^{y} u(x, \tau) d \tau\right)^{2} d x d y \\
-\int_{0}^{b} \int_{0}^{a} B(x, y) u(x, y) \int_{0}^{x} u(t, y) d t d x d y=\frac{1}{2} \int_{0}^{b} \int_{0}^{a} B_{x}(x, y)\left(\int_{0}^{x} u(t, y) d t\right)^{2} d x d y \\
-\int_{0}^{b} \int_{0}^{a} A(x, y) u_{x}(x, y) \int_{0}^{y} u_{x}(x, \tau) d \tau d x d y \\
=\frac{1}{2} \int_{0}^{b} \int_{0}^{a} A_{y}(x, y)\left(\int_{0}^{y} u_{x}(x, \tau) d \tau\right)^{2} d x d y \\
-\int_{0}^{b} \int_{0}^{a} B(x, y) u_{y}(x, y) \int_{0}^{x} u_{y}(t, y) d t d x d y \\
=\frac{1}{2} \int_{0}^{b} \int_{0}^{a} B_{x}(x, y)\left(\int_{0}^{x} u_{y}(t, y) d t\right)^{2} d x d y \\
\int_{0}^{b} \int_{0}^{a} C(x, y) u(x, y) \int_{0}^{y} \int_{0}^{x} u(t, \tau) d t d \tau d x d y \\
=\frac{1}{2} \int_{0}^{b} \int_{0}^{a} C_{x y}\left(\int_{0}^{y} \int_{0}^{x} u(t, \tau) d t d \tau\right)^{2} d x d y \\
-\int_{0}^{b} \int_{0}^{a} C(x, y) \int_{0}^{x} u(t, y) d t \int_{0}^{y} u(x, \tau) d \tau d x d y
\end{gathered}
$$

Теперь разность $B(u, u)-m\|u\|_{1}^{2}$, знак которой нас интересует, можем записать так:

$$
\begin{aligned}
& B(u, u)-m\|u\|_{1}^{2}=\frac{1}{2} \int_{0}^{a} \int_{0}^{b}\left(\left(\frac{M}{2} u^{2}+2 A u u_{y}+2 M u_{y}^{2}\right)+\left(\frac{M}{2} u^{2}+2 B u u_{x}+2 M u_{x}^{2}\right)\right. \\
& \quad+\frac{1}{2}\left(A_{y}\left(\int_{0}^{y} u(x, \tau) d \tau\right)^{2}-2 C \int_{0}^{x} u(t, y) d t \int_{0}^{y} u(x, \tau) d \tau+B_{x}\left(\int_{0}^{x} u(t, y) d t\right)^{2}\right) \\
& \quad+\left(\frac{M}{2} u^{2}-2\left(A_{x}+C\right) u \int_{0}^{y} u_{x}(x, \tau) d \tau+A_{y}\left(\int_{0}^{y} u_{x}(x, \tau) d \tau\right)^{2}\right) \\
& \quad+\left(\frac{M}{2} u^{2}-2\left(B_{y}+C\right) u \int_{0}^{x} u_{y}(t, y) d t+B_{x}\left(\int_{0}^{x} u_{y}(t, y)\right)^{2}\right) \\
& \left.\quad+C_{x y}\left(\int_{0}^{y} \int_{0}^{x} u(t, \tau) d t d \tau\right)^{2}\right) d x d y,
\end{aligned}
$$


и легко видеть, что при выполнении условий леммы

$$
B(u, u)-m\|u\|_{1}^{2} \geqslant 0
$$

откуда и следует справедливость неравенства (5).

Теорема 1. Если выполнены условия леммы 1, то существует не более одного обобщенного решения задачи с условиями (2) для уравнения (4).

ДокАЗАТЕЛЬСтво этого утверждения вытекает из неравенства (5), так как при $F(x, y)=0 B(u, u)=0$, а, стало быть, и $\|u\|_{1}^{2}=0$.

Для доказательства сушествования решения воспользуемся методом Галёркина и будем искать приближенное решение задачи в виде

$$
u_{m}(x, y)=\sum_{k=1}^{m} d_{k}^{m} \Phi_{k}(x, y)
$$

где $\left\{\Phi_{k}(x, y)\right\}$ - базис конечномерного подпространства $V_{m}$ пространства $\widetilde{H}^{1}$, из соотношений

$$
B\left(u_{m}, v_{m}\right)=(F, l v)_{0} .
$$

Равенства (6) представляют собой систему линейных уравнений относительно неизвестных $d_{k}^{m}$ с матрицей $\mathbf{B}=\left(B\left(\Phi_{i}, \Phi_{j}\right)\right)$.

ЛЕмма 2. Система (6) однозначно разрешима.

ДокАЗАТЕЛЬСтво. Однозначная разрешимость этой системы следует из неравенства (5). Действительно, предположим, что существует нетривиальное решение однородной системы, соответствующей системе $(6), z=\left(z_{1}, z_{2}, \ldots, z_{m}\right)$. Рассмотрим функцию

$$
u_{m}^{0}(x, y)=\sum_{k=1}^{m} z_{k} \Phi_{k}(x, y)
$$

Тогда, используя неравенство (5), получим

$$
0=z^{T} \mathbf{B} z=B\left(u_{m}^{0}, u_{m}^{0}\right) \geqslant\left\|u_{m}^{0}\right\|_{1}^{2}
$$

откуда $u_{m}^{0}=0$. В силу линейной независимости функций $\Phi_{k}(x, y)$ имеем $z_{k}=0$ для всех $k=1, \ldots, m$; следовательно, $z=0$.

ТЕОрема 2. Для любой функиии $F(x, y) \in L_{2}(D)$ существует единственная функиия $u_{m}(x, y) \in V_{m}$, удовлетворяющая соотношению (6), причем

$$
\left\|u_{m}\right\|_{1} \leqslant c_{2}\|F\|_{0}
$$

əде $c_{2}=c_{1} \sqrt{3 \max \left\{a^{2} b^{2}, a^{2}, b^{2}\right\}}$. 
ДокАЗАТЕльСтво. Существование и единственность функции $u_{m}(x, y)$, удовлетворяющей соотношению (6), следует из леммы 2. Для доказательства неравенства (7) заметим, что

$$
\begin{aligned}
\|l u\|_{0}^{2} \leqslant & 3 \int_{0}^{b} \int_{0}^{a}\left(\left(\int_{0}^{y} \int_{0}^{x} u(t, \tau) d t d \tau\right)^{2}\right. \\
& \left.+\left(\int_{0}^{y} u_{x}(x, \tau) d \tau\right)^{2}+\left(\int_{0}^{x} u_{y}(t, y) d t\right)^{2}\right) d x d y
\end{aligned}
$$

Применив теперь к каждому слагаемому подынтегрального выражения неравенство Коши-Буняковского, получим

$$
\|l u\|_{0}^{2} \leqslant 3 \int_{0}^{b} \int_{0}^{a}\left(a^{2} b^{2} u^{2}+b^{2} u_{x}^{2}+a^{2} u_{y}^{2}\right) d x d y \leqslant 3 \max \left\{a^{2} b^{2}, a^{2}, b^{2}\right\}\|u\|_{1}^{2} .
$$

Из леммы 1 и полученного неравенства следует

$$
\begin{aligned}
\left\|u_{m}\right\|_{1}^{2} & \leqslant c_{1} B\left(u_{m}, u_{m}\right)=c_{1}\left(F, l u_{m}\right)_{0} \leqslant\|F\|_{0}\left\|l u_{m}\right\|_{0} \\
& \leqslant c_{1} \sqrt{3 \max \left\{a^{2} b^{2}, a^{2}, b^{2}\right\}}\|F\|_{0}\left\|u_{m}\right\|_{1}
\end{aligned}
$$

и, стало быть,

$$
\left\|u_{m}\right\|_{1} \leqslant c_{2}\|F\|_{0}
$$

ТЕОремА 3. Если выполнены условия леммы 1, то для любой функиии $F(x, y) \in$ $L_{2}(D)$ существует обобщенное решение задачи (2)-(4), которое удовлетворяет неравенству

$$
\|u\|_{1} \leqslant c_{2}\|F\|_{0}
$$

ДокАЗАТЕЛЬСТво. Из теоремы 2 следует, что множество функций $u_{m}, m=1,2, \ldots$, ограничено в $\widetilde{H}^{1}(D)$ и, стало быть, слабо компактно, т.е. из него можно выделить подпоследовательность, слабо сходящуюся к некоторой функции $u \in \widetilde{H}^{1}(D)$. Покажем, что эта функция и является решением задачи (2)-(4). Так как каждая из функций $u_{m}(x, y)$ удовлетворяет для любого $i, 1 \leqslant i \leqslant m$, тождеству

$$
B\left(u_{m}, \Phi_{i}\right)=\left(F, l \Phi_{i}\right)
$$

то, умножив каждое из них на $d_{i}$, просуммировав по $i$ от 1 до $m$ и обозначив $\eta_{m}(x, y)=$ $\sum_{i=1}^{m} d_{i} \Phi_{i}(x, y)$, получим

$$
B\left(u_{m}, \eta_{m}\right)=\left(F, l \eta_{m}\right)_{0} .
$$

Переходя в неравенстве (7) и равенстве (8) к пределу при $m \rightarrow \infty$, приходим к утверждению теоремы.

4. Задача для квазилинейного уравнения. Опираясь на полученные в предыдущем пункте результаты для линейного уравнения, докажем разрешимость поставленной задачи. 
ТЕОРемА 4. Пусть $f(x, y, u)$ удовлетворяет условию Каратеодори, а также

a) $\left|f\left(x, y, u_{1}\right)-f\left(x, y, u_{2}\right)\right| \leqslant L_{0}\left|u_{1}-u_{2}\right|$,

б) $|f(x, y, u)| \leqslant \frac{1}{\sqrt{2}}\left(|p(x, y)|+\sqrt{c_{0}^{2}-\eta^{2}}|u|\right)$, где $p(x, y) \in L_{2}(D), 0<\eta \leqslant c_{0}^{2}$, $c_{0}=c_{1}^{-1}$.

Тогда существует по крайней мере одно обобщенное решение $u_{0} \in \widetilde{H}^{1}(D)$ задачи (1), (2) такое, что

$$
\|u\|_{1}^{2} \leqslant \frac{P a b}{\eta^{2}}
$$

где $P=\|p(x, y)\|_{0}$. Если $L_{0}<c_{0}$, то решение единственно.

ДокАЗАтЕЛьСтво. Рассмотрим замкнутый шар

$$
W=\left\{w \mid w \in L_{2}(D),\|w\|_{0}^{2} \leqslant \frac{P^{2} a b}{\eta^{2}}\right\} .
$$

Из условия б) теоремы 4 следует, что для любой $w \in W\|f\|_{0}^{2} \leqslant c_{0}^{2} P^{2} a b / \eta^{2}$. Так как соответствующая линейная задача однозначно разрешима, то можно утверждать, что для любой $w \in W$ существует единственное обобщенное решение задачи

$$
L u=f(x, y, w), \quad \int_{0}^{a} u(x, y) d x=0, \quad \int_{0}^{b} u(x, y) d y=0,
$$

т.е. $B(u, v)=(f(x, y, w), l v)_{0}$ для любой $v \in \widetilde{H}^{1}(D)$, причем $\|u\|_{1}^{2} \leqslant P^{2} a b / \eta^{2}$. Определим оператор $T: w \in W \rightarrow u=T w \in \widetilde{H}^{1}(D), T(W) \subset W$, и покажем, что он вполне непрерьвен.

Условие Липшица а) обеспечивает непрерьвность $T$. Действительно, пусть $\left\{w_{n}\right\}-$ последовательность функций из $W$, сходящаяся к $w_{0} \in W$, т.е. $\left\|w_{n}-w_{0}\right\| \rightarrow 0, n \rightarrow \infty$. Для $u_{n}=T w_{n}$ и $u_{0}=T w_{0}$ имеем

$$
B\left(u_{n}-u_{0}, v\right)=\left(f\left(x, y, w_{n}\right)-f\left(x, y, w_{0}\right), l v\right)_{0} .
$$

Из неравенства (5) и условия а) теоремы 4

$$
\left\|u_{n}-u_{0}\right\|_{1} \leqslant c_{1}\left\|f\left(x, y, w_{n}\right)-f\left(x, y, w_{0}\right)\right\|_{0} \leqslant P c_{1}\left\|w_{n}-w_{0}\right\|_{0}
$$

откуда следует, что $\left\|u_{n}-u_{0}\right\|_{1} \rightarrow 0, n \rightarrow \infty$, и, стало быть, оператор $T$ непрерьвен.

Заметим далее, что для $u_{n}=T w_{n}, w_{n} \in W$, справедливо неравенство $\left\|u_{n}\right\|_{1}^{2} \leqslant$ $P^{2} a b / \eta^{2}$; следовательно, существует подпоследовательность, слабо сходящаяся к некоторому элементу $u_{0} \in \widetilde{H}^{1}$. Так как ограниченное в $H^{1}$ множество компактно в $L_{2}$, $\widetilde{H}^{1}(D) \subset H^{1}(D)$, существует подпоследовательность, которую обозначим также $\left\{u_{n}\right\}$, сходящаяся сильно в $L_{2}$. Тогда $\left\|u_{0}\right\|_{0}^{2} \leqslant P^{2} a b / \eta^{2}$; стало быть, $u_{0} \in W$ и оператор $T$ компактен. В силу теоремы Шаудера о неподвижной точке существует по крайней мере одна точка $u_{0} \in W$ такая, что $u_{0}=T u_{0} \in \widetilde{H}^{1}(D)$ и

$$
B\left(u_{0}, v\right)=\left(f\left(x, y, u_{0}\right), l v\right)_{L_{2}} .
$$

Предположим, что существует два различньх обобщенных решения $u_{1}$ и $u_{2}$. Тогда

$$
B\left(u_{1}-u_{2}, v\right)=\left(f\left(x, y, u_{1}\right)-f\left(x, y, u_{2}\right), l v\right)_{0} .
$$

Из неравенства (5)

$$
\left\|u_{1}-u_{2}\right\|_{0} \leqslant\left\|u_{1}-u_{2}\right\|_{1} \leqslant c_{1}\left\|f\left(x, y, u_{1}\right)-f\left(x, y, u_{2}\right)\right\|_{0} \leqslant L_{0} c_{1}\left\|u_{1}-u_{2}\right\|_{0} .
$$

Если $L_{0} c_{1}<1$, т.е. $L_{0}<c_{0}$, то из последнего неравенства $\left\|u_{1}-u_{2}\right\|_{0}=0$ и, стало быть, решение единственно. 


\section{СПИСОК ЦИТИРОВАННОЙ ЛИТЕРАТУРЫ}

[1] Самарский А. А. О некоторых проблемах современной теории дифференциальных уравнений // Дифференц. уравнения. 1980. Т. 16. №11. С. 1221-1228.

[2] Камынин Л.И.Об одной краевой задаче теории теплопроводности с неклассическими граничными условиями // ЖВМиМФ. 1964. Т. 4. № 6. С. 1006-1024.

[3] Ионкин Н. И. Решение одной краевой задачи теории теплопроводности с неклассическим краевым условием // Дифференц. уравнения. 1977. Т. 13. № 2. С. 294-304.

[4] Cannon J.R. The solution of the heat equation subject to the specification of energy // Quart. Appl. Math. 1963. V. 21. № 2. P. 155-160.

[5] Алексеева С. М., Юрчук Н. И. Метод квазиобращения для задачи управления начальным условием для уравнения теплопроводности с интегральным краевым условием // Дифференц. уравнения. 1998. Т. 34. № 4. С. 495-502.

[6] Bouziani A., Benouar N-E. Problème mixte avec conditions integrales pour une class d'équations paraboliques // C. R. Acad. Sci. Paris Sér. I. 1995. V. 321. P. 1177-1182.

[7] Муравей Л.А., Филиновский А. В. Об одной нелокальной краевой задаче для параболического уравнения // Матем. заметки. 1993. Т. 54. № 4. С. 98-116.

[8] Нахушев А. М. Об одном приближенном методе решения краевых задач для диффференциальных уравнений и его приложения к динамике почвенной влаги и грунтовых вод // Дифференц. уравнения. 1982. Т. 18. №1. С. 72-81.

[9] Водахова В. А. Краевая задача с нелокальным условием А. М. Нахушева для одного псевдопараболического уравнения влагопереноса // Дифференц. уравнения. 1982. Т. 18. № 2. C. $280-285$.

[10] Камынин В. Л., Саролди М. Нелинейная обратная задача для параболического уравнения высокого порядка // ЖВМиМФ. 1998. Т. 38. №10. С. 1683-1691.

[11] Нахушев А. М. Уравнения математической биологии. М.: Высшая школа, 1995.

[12] Белавин И. А., Капица С. П., Курдюмов С. П. Математическая модель глобальных демографических процессов с учетом пространственного распределения // ЖВМиМФ. 1998. T. 38. №6. С. $885-902$.

[13] Гущин А. К.,Михайлов В.П. О разрешимости нелокальных задач для эллиптического уравнения второго порядка // Матем. сб. 1994. Т. 185. №1. С. 121-160.

[14] Панеях Б. П. О некоторых нелокальных краевых задачах для линейных дифференциальных операторов // Матем. заметки. 1984. Т. 35. № 3. С. 425-434.

[15] Скубачевский А. Л., Стеблов Г. М. О спектре дифференциальных операторов с областью определения, не плотной в $L_{2}(0,1)$ // Докл. АН СССР. 1991. Т. 321. №6. С. 1158-1163.

[16] Скубачевский А. Л. О спектре некоторых нелокальных эллиптических краевых задач // Матем. сб. 1982. Т. 117 (159). № 4. С. 548-558.

[17] Bouziani A. Solution forte d'un problème mixte avec conditions non locales pour une classe d'équations hyperboliques // Bull. Cl. Sci. Acad. Roy. Belg. 1997. V. 8. P. 53-70.

[18] Гордезиани Д. Г., Авалишвили Г. А. Решения нелокальных задач для одномерных колебаний среды // Матем. моделирование. 2000. Т. 12. №1. С. 94-103.

[19] Пулькина Л. С. О разрешимости в $L_{2}$ нелокальной задачи с интегральными условиями для гиперболического уравнения // Дифференц. уравнения. 2000. Т. 36. № 2. С. 279-280.

[20] Нахушева З. А. Об одной нелокальной задаче для уравнений в частных производных // Дифференц. уравнения. 1986. Т. 22. №1. С. 171-174.

Самарский государственный университет 